\title{
Rotational remagnetization and orientational transitions in submicron YIG films
}

\author{
Poimanov Vladyslav ${ }^{1, *}$, Nepochatykh Yurii ${ }^{2}$, Koledov Victor $^{3}$, Shavrov Vladimir ${ }^{3}$ \\ ${ }^{1}$ Donetsk National University, 83001, Universitetskaya 24, Donetsk, Ukraine \\ ${ }^{2}$ Donetsk institute for physics and engineering named after A.A.Galkin, 83001, R. Luxemburg str. 72, Donetsk, Ukraine \\ ${ }^{3}$ Kotel'nikov institute of radio engineering and electronics of RAS, 125009, Mokhovaya str., Moscow, Russia
}

\begin{abstract}
It is shown that for the quasinormal remagnetization of the submicron YIG films with the normal along the [111] axis, which are in the single-domain state, two types of orientation transitions (OT) due to the influence of easy axes can be distinguished. The nonreciprocity of the FMR spectrum for forward and reverse magnetization due to the influence of easy axes is shown.
\end{abstract}

\section{Introduction}

At present a hypothesis exists that submicron ferritegarnet film (thickness $\sim 0.1 \mu \mathrm{m}$ ), obtained by the twocrucible liquid-phase epitaxy technique from wetting melt-solution on a nonmagnetic substrate [1-2] are in a single-domain state. This opens the prospect of their use as both switching devices and high frequency field generators that do not require the presence of a permanent magnet, which can significantly reduce the weight of the device.

The results of numerical modeling of the remagnetization of these films by a quasinormal field are presented. In the experiment, it is quite difficult to ensure that the external field is applied strictly normal to the film. This also concerns the orientation of the easy axes of cubic symmetry - the growth of the film can occur not strictly along any axis of symmetry. Due to the asymmetry in the plane of the film this leads to a number of interesting effects. In particular, there appears a nonreciprocity of direct and reverse remagnetization both for the angular dependences of the dynamic susceptibility and for the frequency field dependencies.

\section{Analysis of expression for density of energy}

Taking into account all types of interactions (cubic, magnetodipole, Zeeman and unidirectional), the density of magnetic energy has the form [3]:

$$
\begin{aligned}
& W=4 \pi M^{2} \frac{\cos ^{2} \theta}{2}-H_{C} M\left(\frac{\sin ^{4} \theta}{4}+\frac{\cos ^{4} \theta}{3}+\frac{\sqrt{2}}{3} \sin ^{3} \theta \cos \theta \cos (3 \varphi)\right) \\
& -H M\left(\cos \theta \cos \theta_{H}+\sin \theta \sin \theta_{H} \cos \left(\varphi-\varphi_{H}\right)\right)-H_{b} M \cos \theta
\end{aligned}
$$

where $\theta$ and $\varphi$ - polar and azimuth angles of orientation of equilibrium orientation of magnetization, $\theta_{H}$ and $\varphi_{H}-$ angles of external field orientation.

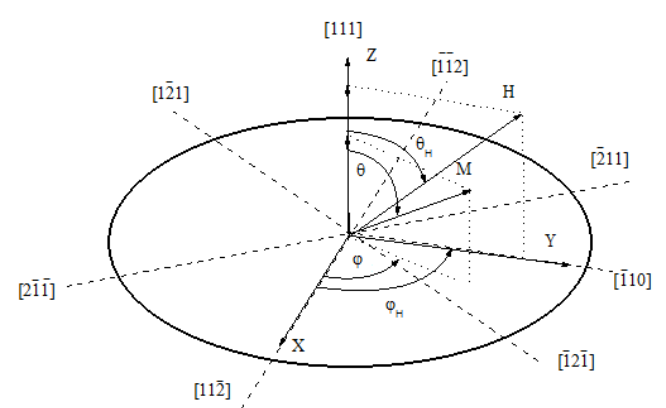

Fig. 1. Geometry of the problem.

Since the cubic anisotropy field $H_{C}=45$ Oe is much smaller than the saturation magnetization $4 \pi M=$ $1250 \mathrm{Oe}$, then there are two types of orientational transition (OT). The first one of them (OT1) is observed in external fields of the order of the anisotropy field $H_{C}$, when the magnetization $M$ is quasi-planar - the exit angle from the plane in the film is less than $1 \mathrm{deg}$. Accordingly, the contribution to the total energy from the demagnetizing field of the film $\left(4 \pi M^{2}\right)$ is comparable with the cubic anisotropy energy $\left(\sim H_{C} M\right)$. In such fields, the main role is played by OT with rotation in the plane of the film (rotational remagnetization, RR), while the normal component of $M$ varies weakly. To suppress the normal component and subsequent reorientation to a new metastable state, it is sufficient to apply a small (to the extent of smallness of the constants of the cubic and unidirectional anisotropy) negative field, the value of which can be obtained from (1) $H_{1}=0.47 H_{C}=20$ Oe. This shows the possibility of controlling a relatively small field along the normal by the magnetization in the plane.

The second type OT (OT2) is associated with remagnetization along the normal and occurs already in

* Corresponding author: Vladislav.Poymanow@yandex.ru 
another interval of external fields (order of $4 \pi M$ ). In this case, on the contrary, the orientation of $M$ in the plane varies weakly, to the extent of the smallness of the planar component of the field $H_{\perp}$. The role of cubic anisotropy (CA) in this case is to the fact $M$ is "fixed" in one of the "easy" directions and, with an increase in the field, without hysteresis, it reaches the normal. The planar field $H_{\perp}$ plays the role of a perturbation and leads to a nonreciprocity of the oscillation spectrum with respect to the direction of the field.

\section{Results of numerical modeling of the remagnetization process}

The results of modeling on the basis of $W$ are given below.
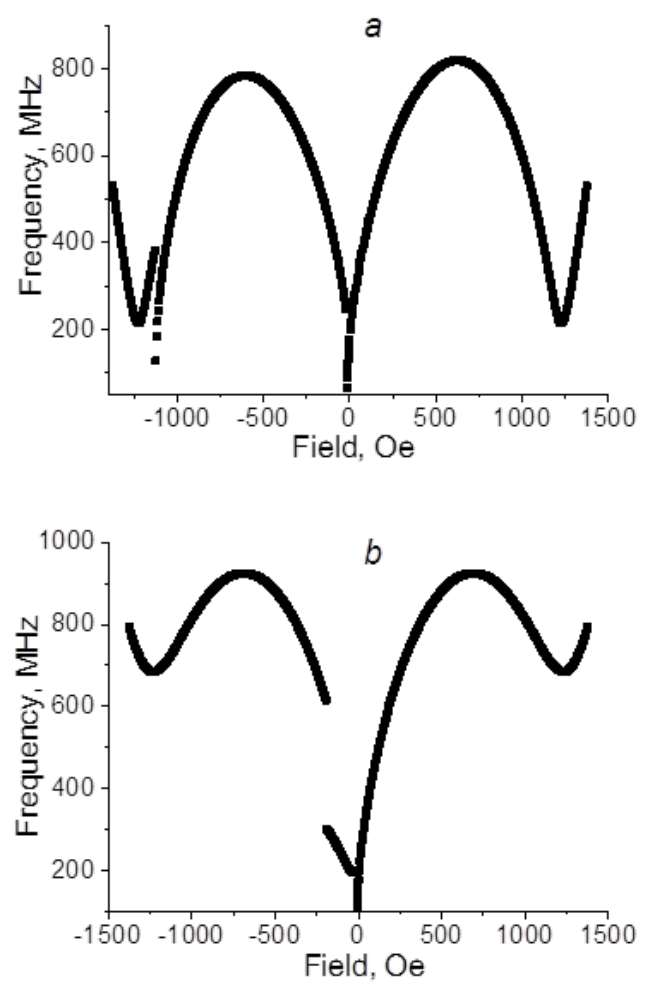

Fig. 2. Frequency-field dependencies for change of the field from -1400 Oe to 1400 Oe in the direction $\varphi_{H}=30$ deg., $\theta_{H}=0.5$ deg.(a) and $\theta_{H}=3$ deg.(b)

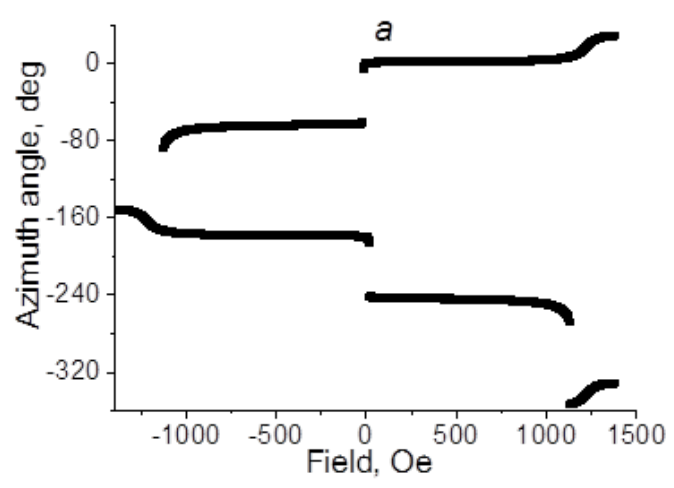

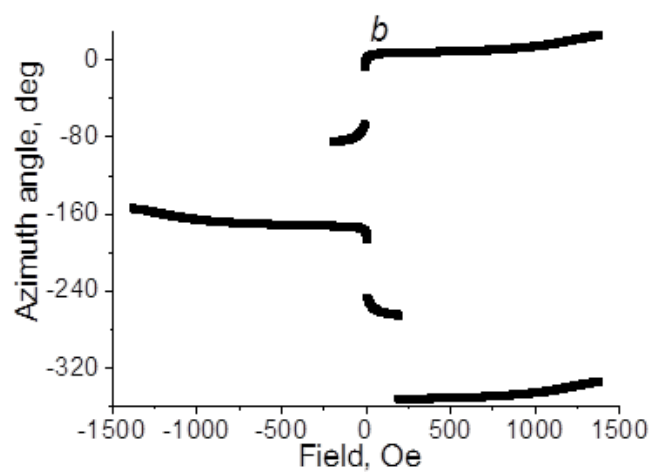

Fig. 3. Rotation of the magnetization in azimuth during one cycle of remagnetization 0 Oe $\rightarrow-1400$ Oe $\rightarrow 1400$ Oe $\rightarrow$ 0 Oe in the direction $\varphi_{H}=30 \mathrm{deg}$. $\theta_{H}=0.5 \mathrm{deg}$. (a) and $\theta_{H}=3 \mathrm{deg}$. (b)

The gaps on the dependencies caused by orientational transitions. As $\theta$ increases, the orientational transition near the saturation field vanishes, and the gap near OT1 increases.

With a full cycle of remagnetization, the magnetization in the plane passes a complete counter-clockwise rotation. This behavior is typical for the azimuthal angles of the application of the field $0 \prec \theta_{H} \prec \frac{\pi}{2}, \frac{2 \pi}{3} n \prec \varphi_{H} \frac{\pi}{3}+\frac{2 \pi}{3} n$. The direction of rotation is reversed at $0 \prec \theta_{H} \prec \frac{\pi}{2}, \frac{\pi}{3}+\frac{2 \pi}{3} n \prec \varphi_{H} \frac{2 \pi}{3}+\frac{2 \pi}{3} n$. This effect can be used in the device for switching the direction of rotation. The polar angle is weakly sensitive to the presence of easy axes, and for $\theta_{H}>1^{\circ}$ the dependence $\theta(H)$ is extrapolated well by the straight line.
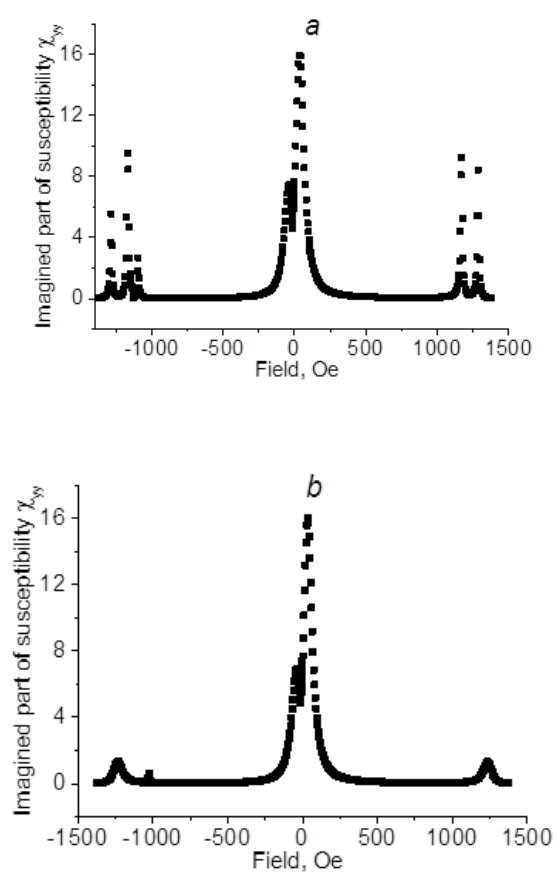

Fig. 4. Dynamic susceptibility $\chi_{y y}$ at frequency $300 \mathrm{MHz}$ for $\varphi_{H}=30$ deg., $\theta_{H}=0.5$ (a) and $\theta_{H}=3$ deg.(b) 
As follows from the shown dependencies if the angle of application of the field deviates from the normal, the value of the field OR2 shifts toward smaller negative fields, which is associated with the increasing role of the planar component. In this case, the gap in the spectrum of the frequency-field dependencies also increases, as evidenced by the disappearance of lateral peaks of susceptibility (fig. 4). For $\theta_{H}=0.5^{\circ}$ there are six distinct peaks arranged symmetrically in the field, and already for $\theta_{H}=1^{\circ}$ this symmetry is violated, since OT2 is expressed more clearly.

\section{Accounting of unidirectional anisotropy}

In the presence of unidirectional anisotropy (UA), in addition to the expected shift of the curve by the value of the field UA, an additional asymmetry also arises. In particular, now the size of the gaps for the positive and negative fields is different. If, without UA, $\Delta v=337$ $\mathrm{MHz}$, then, if it is present, $\Delta v_{-}=343.4 \mathrm{MHz}, \Delta v_{+}=$ 330.7 MHz.

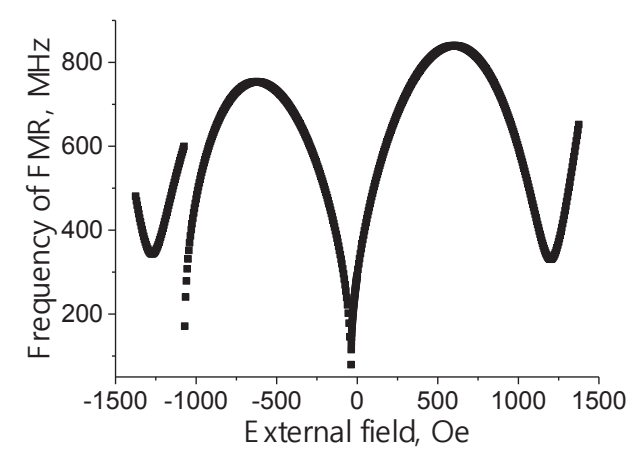

Fig. 5. Frequency-field dependency with presence of unidirectional anisotropy $\varphi_{H}=30 \mathrm{deg}$. $\theta_{H}=1 \mathrm{deg}$.

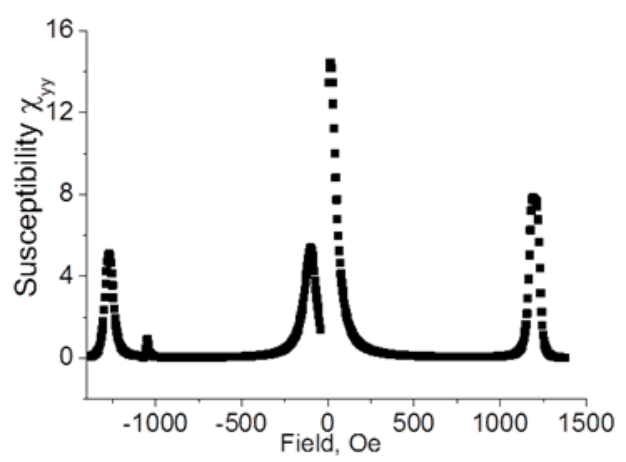

Fig. 6. Asymmetry of the susceptibility peaks for the frequency of $332 \mathrm{MHz}$, due to different lower FMR frequencies for direct and reverse magnetization.

The simulation data of the frequency-field dependencies for different $\varphi_{H}$ show that the lower frequency limit of the frequency-field dependencies is significantly reduced when rotating in azimuth from 0 to 60 degrees. The field corresponding to the minimum frequency shifts toward decreasing with increasing azimuth angle from 0 to 60 degrees, and this shift is the larger, the larger $\theta_{H}$.

\section{Influence of azimuth angle of the applied field $\varphi_{H}$}

When the film is rotated along the azimuth, the lower frequency limit of the FMR (gap) changes, reaching the smallest value at $\varphi_{H}=60^{\circ}$. The effect of unidirectional anisotropy turns to the asymmetry of FMR observation at a given frequency.

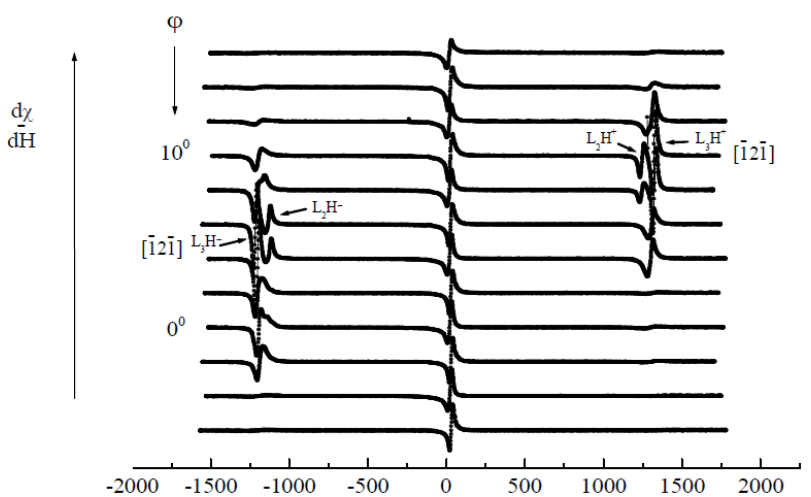

Fig. 7. Variation of the derivative of magnetic susceptibility with quasinormal external $\left(\theta_{H}=1 \mathrm{deg}\right.$.) field [4] for a range of azimuthal angles $\left(\varphi_{H}\right)$ separated by an interval of $2 \mathrm{deg}$. $(v=$ $300 \mathrm{MHz}$ at room temperature).

\section{Conclusions}

1. The presence of an angle of misorientation of the applied field from the normal to the film [111] leads to the appearance of orientational transitions of two types. The first of them occurs in a field of the order of cubic anisotropy, the second - in a field of the order of saturation magnetization.

2. The possibility of controlling the rotation of the magnetization in the plane of the film is shown.

3. With an increase in the polar angle of application of the field to the normal, the value of the gap in the spectrum of the frequency-field dependency increases, and the field OT2 decreases in absolute value.

4. The presence of unidirectional anisotropy, in addition to the shift of the frequency-field dependency, leads to an additional asymmetry in the FMR spectrum - the sizes of the gaps for direct and reverse magnetization turn out to be different.

This work was supported by Russian Science Foundation, grant No. 14-22-00279

\section{References}

[1] V.F.Shkar', V.N.Varyukhin, JETP Letters, 88 (2008)

[2] V.F.Shkar', V.N.Varyukhin, JETP Letters 92 (2010)

[3] A.G. Gurevich, Magnetic Resonance in Ferrites and Antiferrornagnets [in Russian] (Nauka, Moscow, 1973)

[4] V. Poimanov, V. Shkar, Yu. Nepochatykh al. JMMM, 443 (2017) 